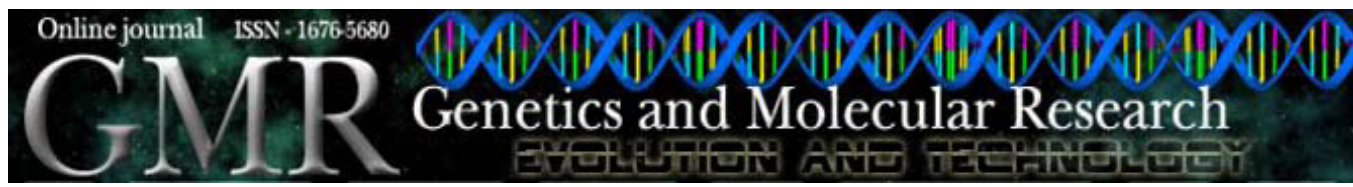

\title{
Biodiversity in the sorghum (Sorghum bicolor L. Moench) germplasm of Pakistan
}

\author{
A. Iqbal', B. Sadia', A.I. Khan', F.S. Awan', R.A. Kainth ${ }^{3}$ and \\ H.A. Sadaqat ${ }^{2}$ \\ ${ }^{1}$ Centre of Agricultural Biochemistry and Biotechnology, \\ University of Agriculture, Faisalabad, Pakistan \\ ${ }^{2}$ Department of Plant Breeding and Genetics, \\ University of Agriculture, Faisalabad, Pakistan \\ ${ }^{3}$ Fodder Research Substation, Ayub Agricultural Research Institute, \\ Faisalabad, Pakistan \\ Corresponding author: B. Sadia \\ E-mail: bushrauaf@gmail.com
}

Genet. Mol. Res. 9 (2): 756-764 (2010)

Received December 16, 2009

Accepted January 18, 2010

Published April 27, 2010

DOI 10.4238/vol9-2gmr741

\begin{abstract}
Sorghum ranks fifth in worldwide economic importance among cereal crops and is one of the most important summer annual grasses of Pakistan. As it is a very diverse crop, sorghum genetic fingerprinting requires an efficient marker system. We estimated genetic divergence among 29 sorghum (Sorghum bicolor) genotypes, including approved varieties and local and exotic lines collected from different ecological regions of Pakistan, using random amplified polymorphic DNA (RAPD) markers. A total of 125 RAPD loci, with an average of 66 loci per genotype, were used to calculate genetic divergence among these genotypes, of which 119 were polymorphic, showing $95 \%$ overall polymorphism. Genetic similarity ranged from 0.36 to 0.92 , indicating a relatively broad genetic base. RAPD analysis revealed maximum similarity between the Indian III and K-A-113 sorghum genotypes (both exotic lines), while the F-601 and F-606 were observed to be the most diverse genotypes. Mean band frequency revealed by these RAPD primers ranged from 0.17 to 0.56 , with an average of 0.36 . The data presented here support the findings that RAPDs can be effectively used
\end{abstract}


for studying genetic diversity in sorghum.

Key words: Sorghum bicolor; RAPD; Genetic diversity; Biofuels; Fodder

\section{INTRODUCTION}

Sorghum (Sorghum bicolor L. Moench) ranks fifth in worldwide economic importance among cereal crops with an annual production of 60 million tons. Besides being an important food, feed and forage crop, sorghum also provides raw material for the production of starch, fiber, dextrose syrup, biofuels, alcohol, and other products. More than half of the world's sorghum is grown in semi-arid tropics of India and Africa, where it is a staple food for millions of poor people (Mehmood et al., 2008). Classical plant breeding has resulted in the successful development of high yielding, highly adapted sorghum cultivars. However, it has some inherent limitations due to natural sexual incompatibility barriers and the narrow genetic variability in sorghum. Therefore, further enhancement of productivity, quality and resistance to biotic and abiotic stresses needs urgent attention.

Plant biotechnology is a promising tool for changing agriculture, potentially providing new solutions to age-old agricultural problems. However, not much has been invested in sorghum in the past as in other cereals, and few laboratories in the world are involved in sorghum crop improvement programs. Since, sorghum has long been considered the most recalcitrant crop for biotechnological applications, owing to the release of phenolics, lack of regeneration in long term in vitro cultures, and a high degree of genotype dependence (Visarada and Kishore, 2007).

In recent years though, growing interest in sorghum due to its small genome size and potential as a biofuel crop has secured a promising place for this crop in global agricultural improvement plans (Paterson et al., 2004). To date, various biotechniques, including tissue culture (Baskaran and Jayabalan, 2005), genetic transformation (Godwin and Seetharama, 2005), molecular markers, genomics, proteomics and tilling, among others, have been successfully exploited in sorghum (Dillon et al., 2005). The sorghum genome has recently been sequenced, providing a greater understanding of genomics-assisted breeding in this crop (Paterson et al., 2009).

In Pakistan, sorghum is cultivated for grain and fodder purposes on an area of 250 thousand hectares (Anonymous, 2008), where it is preferred as feed. Shortages of forage crops in Pakistan most often prevail twice a year (May-June and October-November). Being one of the most important summer annual grasses of Pakistan, sorghum improvement for yield and quality characters can greatly reduce the risk of inadequate forage production during summer.

Plant genetic resources play an important role in generating new high yielding crop varieties with resistance to biotic and abiotic stresses (Murray et al., 2008). Morphological, biochemical and molecular procedures have been exploited for evaluating these resources. Until recently, either qualitative or quantitative morphological characters were exploited for most of the crop characterization and evaluation. However, phenotypic variation is not truly indicative of genetic variation due to environmental interaction in the resultant phenotype. Biochemical markers on the other hand reflect more truly the genetic variability, as they are the direct products of genes (Romero et al., 2009). Genetic fingerprinting is one of the DNAbased techniques with various applications in crop biology including assessment of taxonomy and phylogeny, diversity analysis, hybridity testing, gene mapping, molecular breeding, and 
somaclonal variation (Mehmood et al., 2008). Among agricultural crops, rice and maize are the most intensively characterized for DNA markers (Romero et al., 2009).

So far, sorghum germplasm improvement strategies in Pakistan have been based only on traditional breeding. Currently, the genetic base within Sorghum bicolor, a species with the greatest share in commercial sorghum varieties, has narrowed over time because of the normal breeding process. Under the present situations, superior genetic resources and technology are required to improve yield and reduce the risk of loss from biotic and abiotic stresses in sorghum.

Sorghum is a very diverse crop and studying the genetic diversity of sorghum germplasm collections from Pakistan attracts special interest. Besides being an important economic crop, a large amount of genetic variation is present in cultivated and wild sorghum germplasm of Pakistan. However, the genetic diversity of available sorghum germplasm of Pakistan has not been fully investigated. Hence, the present research is a pioneering attempt at estimating the biodiversity of 29 sorghum genotypes from all over Pakistan, using 32 random amplified polymorphic DNA (RAPD) markers.

\section{MATERIAL AND METHODS}

\section{Plant material}

The plant material comprised 29 sorghum genotypes, including 3 varieties, 6 exotic lines and 20 local lines, from the different ecological background of Pakistan; their salient morphological traits are described in Table 1.

\begin{tabular}{|c|c|c|c|}
\hline Series No. & Name & Parentage & Key trait \\
\hline 1 & Hegari & Variety & Tall, juicy, high yielder, multi-cut \\
\hline 2 & Noor & JS-2002 × F-507 & Medium, moderate juicy, multi-cut \\
\hline 3 & JS-2002 & Variety & Tall, sweet, small head \\
\hline 4 & F-507 & Sild marhar $\times$ Indian III & Medium, less sweet, seed yield high \\
\hline 5 & F-603 & JS-88 $\times$ Sorokarthahu & Tall, leafy, good seed yield, sweet \\
\hline 6 & F-505 & Pak-47 $\times$ China small & Medium, leafy, light sweet, more fodder \\
\hline 7 & $\mathrm{~F}-215$ & JS- $88 \times$ Australian- 6 & Medium, sweet, leafy \\
\hline 8 & FJ-9601 & Hegari $\times$ Pak 47 & Tall, sweet, good palatability \\
\hline 9 & F-9809 & Sorkarthahu $\times$ JS-263 & Tall, leafy, less sweet, more fodder \\
\hline 10 & F-502 & Sugrorib $\times$ Australian-7 & Short internode, sweet, leafy \\
\hline 11 & F-9917 & Sugrorib $\times$ JS-2002 & Tall, sweet, large head size \\
\hline 12 & F-2012 & Australian Sudan grass $\times$ Tandi & Multi-cut, leafy, good fodder yield \\
\hline 13 & F-9606 & JS-263 × BJ-1914 & Less sweet, good fodder yield, lush green \\
\hline 14 & F-2022 & $\mathrm{BJ}-20 \times \mathrm{JS}-88$ & Medium, less sweet, large head size \\
\hline 15 & $\mathrm{~F}-214$ & JS-2002 $\times$ China small & Medium, sweet, juicy, good yield \\
\hline 16 & F-2020 & Tandi $\times$ A82L-36 & Medium, sweet, erect, good yield \\
\hline 17 & F-2018 & JS-2002 $\times$ Sorokarthahu & Tall, lush green, sweeter, good yield \\
\hline 18 & F-9706 & Hegari $\times$ JS-263 & Tall, less sweet, narrow leaves \\
\hline 19 & F-9603 & Australian-7 $\times$ JS-263 & Medium, greenish midrib, good yield \\
\hline 20 & F-604 & $\mathrm{JS}-88 \times \mathrm{BJ}-20$ & Tall, compact head, less sweet \\
\hline 21 & F-601 & Succro $\times$ Sudan grass & Multi-cut, medium, juicy, good yield \\
\hline 22 & F-508 & Indian III $\times$ Sildmarhar & Medium, less sweet, small head \\
\hline 23 & F-606 & Sugrorib $\times$ China small & Juicy, medium to tall, waxy, broad leaves \\
\hline 24 & Indian III & Exotic line & Medium, loose head size, broad leaves \\
\hline 25 & Australian-7 & Exotic line & Medium, leafy, short internodal distance \\
\hline 26 & $\mathrm{~K}-\mathrm{A}-113$ & Exotic line & Short, large head size, grain red color \\
\hline 27 & Australian-6 & Exotic line & Bold grain size, short, sweet \\
\hline 28 & China small & Exotic line & Medium to tall, erect, sweet \\
\hline 29 & A82L-36 & Exotic line & Bold grain size, short, sweet \\
\hline
\end{tabular}




\section{DNA extraction}

Fresh leaf samples were collected from 10-day-old seedlings, and genomic DNA was extracted from one or more individual seedlings from each genotype following a modified CTAB method (Khan et al., 2004). The extracted DNA was electrophoresed on a $0.8 \%$ agarose gel for quality assessment and quantified spectrophotometerically (CECIL, CE 20212000 Series, Cambridge, UK) at $260 \mathrm{~nm}$.

\section{DNA amplification by RAPDs}

The polymerase chain reaction (PCR) was run in a final volume of $25 \mu \mathrm{L}$ containing $2.5 \mu \mathrm{L}$ DNA template, $0.2 \mu \mathrm{L}$ Taq DNA polymerase (MBI, Fermentas, Vinius, Lithuania), 2.5 $\mu \mathrm{L} 10 \mathrm{X}$ buffer, $2.5 \mu \mathrm{L}$ gelatin, $3.0 \mu \mathrm{L} \mathrm{MgCl}_{2}, 4.0 \mu \mathrm{L}$ dNTPs, $2.0 \mu \mathrm{L}$ RAPD primer and 8.3 $\mu \mathrm{L} \mathrm{d}_{3} \mathrm{H}_{2} \mathrm{O}$. The amplifications were performed in a thermal cycler (AG No. 533300839, Germany), following the program: $95^{\circ} \mathrm{C}$ for $5 \mathrm{~min}, 45$ cycles $\left(1 \mathrm{~min}\right.$ at $95^{\circ} \mathrm{C}$, at $34^{\circ} \mathrm{C}$ for $1 \mathrm{~min}$, at $72^{\circ} \mathrm{C}$ for $2 \mathrm{~min}$ ) and final elongation of $10 \mathrm{~min}$ at $72^{\circ} \mathrm{C}$.

Four series of RAPD primers (A, C, J, and K) custom synthesized from Genelink, were used for RAPD analysis, and 32 polymorphic primers were selected to amplify the genomic DNA of sorghum germplasm (Table 2).

\begin{tabular}{lcc}
\multicolumn{2}{c}{ Table 2. RAPD primers along with their sequence, used in the study. } & \\
\hline Series No. & Primer name & Sequence \\
\hline 1 & GLA-04 & AATCGGGCTG \\
2 & GLA-05 & AGGGGTCTTG \\
3 & GLA-06 & GGTCCCTGAC \\
4 & GLA-08 & GTGACGTAGG \\
5 & GLA-12 & TCGGCGATAG \\
6 & GLA-13 & GGCACCCAC \\
7 & GLA-17 & GACCGCTTGT \\
8 & GLA-20 & GTTCGATCC \\
9 & GLC-01 & GGGAGCCAG \\
10 & GLC-03 & GGGGCTTT \\
11 & GLC-07 & TGGCGCGA \\
12 & GLC-08 & AAAGCTGG \\
13 & GLC-11 & TGTCATCCCC \\
14 & GLC-12 & AAGCCTCGTC \\
15 & GLC-13 & TGCGTGCTTG \\
16 & GLC-14 & CACACTCCAG \\
17 & GLC-16 & GTTGCCAGCC \\
18 & GLC-19 & ACTTCGCCAC \\
19 & GLC-20 & TGAGCCTCAC \\
20 & GLJ-09 & ACTCCTGCGA \\
21 & GLJ-11 & GTCCGTGGT \\
22 & GLJ-12 & CCACACTACC \\
23 & GLJ-13 & TGGTCGCAGA \\
24 & GLJ-18 & GGACACCACT \\
25 & GLJ-19 & AAGCGGCCTC \\
26 & GLJ-20 & CCGCCCAAAC \\
27 & GLK-04 & AGCGAGCAAG \\
28 & GLK-07 & GTGCAACGTG \\
29 & GLK-10 & AATGCCCCAG \\
30 & GLK-11 & TGGCCCTCAC \\
31 & GLK-12 & GTGTCGCGAG \\
32 & GLK-20 &
\end{tabular}

RAPD amplification products were analyzed by electrophoresis on $1.2 \%$ agarose gels, run in $1 \mathrm{X}$ TBE, visualized under a UV transilluminator, and photographed using SyneGene gel documentation system. The data generated from the detection of polymorphic fragments 
were analyzed using the Popgen32 software (version 1.44) (Yeh et al., 2000). All amplification products of each RAPD marker were scored as " 1 " or " 0 ", where " 1 " indicated the presence of a specific allele (band) and " 0 " indicated its absence. Multivariate analysis was conducted to generate a similarity matrix using the Popgen32 software, version 1.44 (Yeh et al., 2002) based on Nei's unweighted pair-group method with arithmetic average (UPGMA) to estimate genetic distance and relatedness of sorghum germplasm.

\section{RESULTS}

\section{Band scoring and cluster analysis}

A total of 125 bands were amplified in the PCR of 29 genotypes, of which 119 were polymorphic, showing $95 \%$ of overall polymorphism. The number of bands produced per genotype ranged from 35 to 90 with an average of 66 bands per genotype. Sorghum line F-502 produced the maximum number of bands while F-2018 gave the minimum number of bands (Figure 1). The low degree of similarity indicated high divergence between the genotypes. The number of amplification products produced per primer varied between 4 and 12 with an average of 7.1 bands per primer.

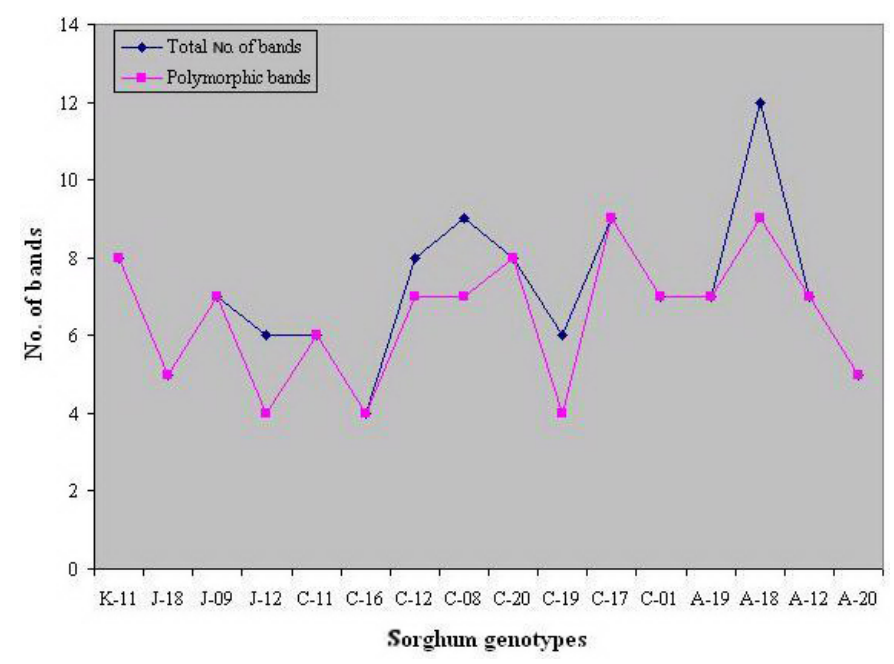

Figure 1. Comparison of sorghum genotypes based on the number of polymorphic bands per primer.

The maximum number of fragments was produced by the primer GLA-18 (12), while the lowest number of fragments was observed with the primer GLC-16 (4). Mean band frequency per primer ranged from 0.17 (GLA-19) to 0.56 (GLC-20), with an average of 0.36 bands (Table 3). Primer GLC-20 gave the maximum mean band frequency while primer GLA19 produced the minimum band frequency. The dendrogram drawn for the genetic distances is shown in Figure 2. The maximum similarity was observed between the Indian III and Australian-7 genotypes (91\%), while the most diverse genotypes were Hegari and A82L-36 (32.97\%). 
Table 3. Mean band frequency of sorghum genotypes.

\begin{tabular}{lccc}
\hline Primer name & Total No. of bands & Polymorphic bands & Mean band frequency \\
\hline GLA-20 & 5 & 4 & 0.32 \\
GLA-12 & 7 & 6 & 0.19 \\
GLA-18 & 11 & 10 & 0.29 \\
GLA-19 & 7 & 6 & 0.56 \\
GLJ-09 & 7 & 7 & 0.35 \\
GLJ-18 & 5 & 5 & 0.44 \\
GLK-11 & 8 & 7 & 0.31 \\
GLC-01 & 7 & 7 & 0.51 \\
GLC-17 & 9 & 9 & 0.32 \\
GLC-19 & 6 & 8 & 0.39 \\
GLC-20 & 8 & 8 & 0.17 \\
GLC-8 & 9 & 8 & 0.33 \\
GLC-12 & 8 & 4 & 0.56 \\
GLC-16 & 4 & 6 & 0.17 \\
GLA-20 & 6 & 4 & 0.33 \\
\hline
\end{tabular}

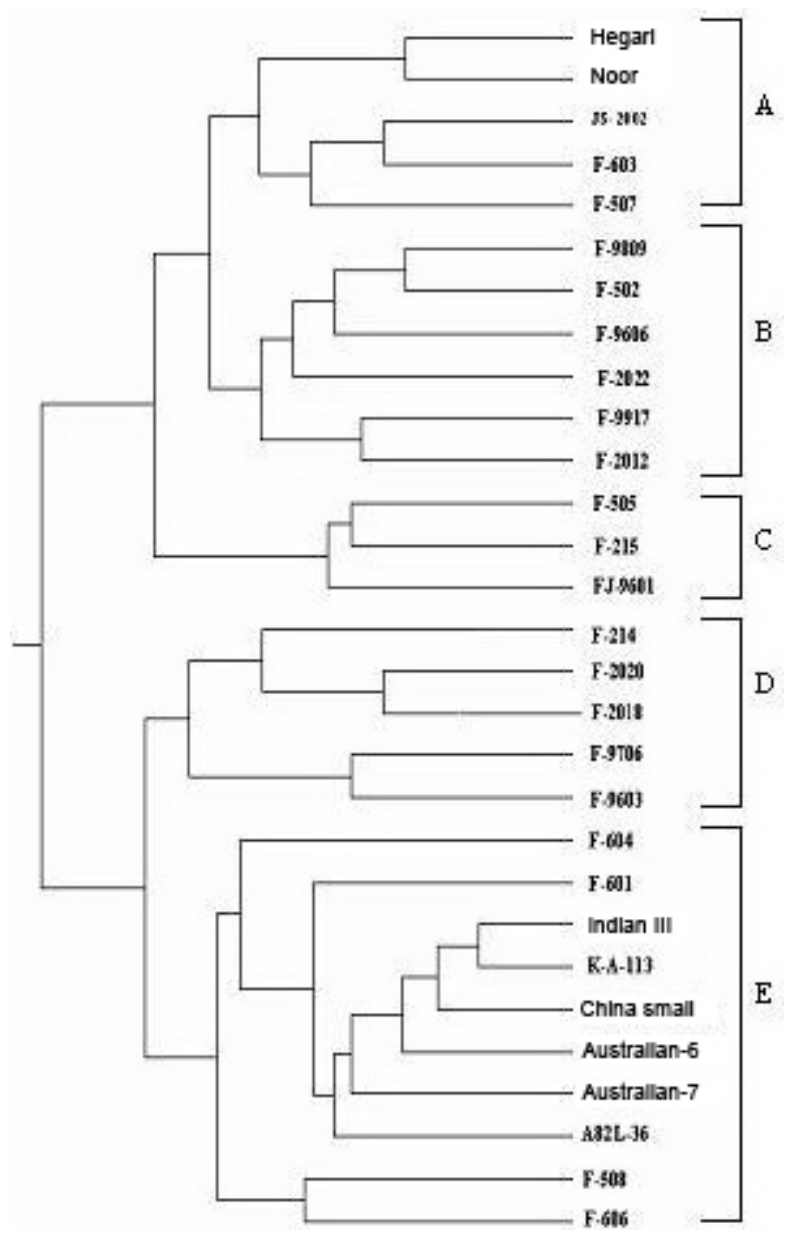

Figure 2. UPGMA dendogram with all tested primers showing the pairwise similarity values for 29 sorghum genotypes. 
Cluster analysis defined two main groups, which were further classified into 5 distinct groups: group A consisted of 5 genotypes, namely Hegari, Noor, JS-2002, F-603, and F-507, showing close similarity among them. In group A, JS-2002, F-507 and F-603 were clustered together while the other two genotypes, Hegari and Noor, remained unclustered, showing a distinct behavior compared to the rest of the group A genotypes. Six sorghum strains, namely F-9809, F-502, F-9606, F-2022, F-9917, and F-2012, were grouped into group B. In this group, F-9809, F-502, F-9606, and 2022 were clustered together, while F-9917 and F-2012 remained unclustered, showing a distinct behavior compared to the rest of the group members. Group C comprised 3 sorghum genotypes, namely F-505, F-215 and FJ-9601. In this group, 2 genotypes (F-505 and F-215) were clustered together, leaving FJ-9601 unclustered. Group D comprised 5 genotypes, namely F-214, F-2020, F-2018, F-9706, and F-9603, wherein F-214, F-2020 and F-2018 were clustered together, while F-9706 and F-9603 showed a distinct behavior compared to other strains of group $\mathrm{D}$, which remained unclustered. The largest group in the dendrogram was group E, with 10 genotypes F-604, F-601, Indian III, K-A-113, China small, Australian-6, Australian-7, A82L-36, F-508, and F-606. Here, F-604, F-601 were grouped together. All the exotic lines, namely Indian III, K-A-113, China small, Australian-6, Australian-7, and A82L-36 were clustered together, while F-508 and F-606 remained unclustered, showing distinct behavior compared to the rest of the genotypes in this group. F-601 and F-604 also showed similarity with the exotic lines clustering in this group.

\section{DISCUSSION}

Plant genetic resources are vital for generating improved crop varieties with resistance to biotic and abiotic stresses. Earlier studies have been mostly devoted to assessing patterns of sorghum genetic variation based on morphology (Djè et al., 1998) or pedigree (Jordan et al., 1998). As sorghum germplasm is very diverse, the assessment of this diversity at the genotypic level requires an efficient marker system. The development of molecular techniques has resulted in alternative DNA-based procedures for detecting polymorphism. Various DNA-based molecular markers have been utilized for analysis of genetic divergence and characterization of sorghum germplasm including RAPDs (Ayana et al., 2000; Agrama and Tuinstra, 2003; Nkongola and Nsapato, 2003; Rajkumar and Kuruvinashetti, 2007; Mehmood et al., 2008; Amrapali et al., 2008), SSRs (Kamala et al., 2006; Dhillon et al., 2006; Perumal et al., 2007; Mohamed et al., 2008), SNPs (Mohamed et al., 2008), AFLPs (Perumal et al., 2007), and ISSRs (Godwin et al., 1997). However, little has been documented on the genetic relationships and diversity within the Pakistani sorghum germplasm. To date, there has been only one account of the application of molecular markers in Pakistani sorghum, specifically of 10 genotypes (Mehmood et al., 2008). One limitation of this previous study was that the sorghum genotypes were fingerprinted only from the Barani tract of Pakistan. Hence, a pioneering attempt was made in the present investigation to fingerprint a large sorghum germplasm from various ecological zones of Pakistan.

The polymorphism percentage observed in the present study was higher than that obtained by Mehmood et al., 2008 (78.94\%). While it was comparable with various reports of sorghum genetic diversity estimates using RAPDS, including Tao et al. (1994), Agrama and Tuinstra (2003) and Nkongola and Nsapato (2003) with 55, 58, and 52\% polymorphism, respectively. The present study indicates higher similarity among sorghum local lines and varieties. Similarly, the exotic sorghum material also showed a lower divergence. However, the low 
degree of similarity (monomorphic bands) was observed between the local and exotic sorghum genotypes, which can be exploited in the selection of crossing parents for sorghum variety development program. The average number of amplification products produced per primer was 9.5, which is higher than that in earlier reports (Amrapali et al., 2008; Mohamed et al., 2008) where mean values were 6.5 and 5.8, respectively. No association could be established between the phenotypic traits and the RAPD markers (except for the two approved varieties Hegari and Noor, clustered in one group, both having the multi-cut trait). Our results are in agreement with an earlier report by Ayana et al. (2000) who observed no genetic relationship (allozyme plus RAPD) with quantitative agro-morphological traits, which shows no correlation between molecular markers and morphological traits. Likewise, an insufficient relationship between RAPD markers and agronomic descriptors was also reported by Dahlberg et al. (2002). This may be due to the influence of different environmental conditions on the phenotypic traits, leading to apparent differences even among identical genotypes (Shehzad et al., 2009).

\section{CONCLUSION}

In conclusion, our results demonstrate that RAPD analysis can be efficiently applied to assess the genetic diversity and phylogenetic relationship of sorghum germplasm. However, there is still a need to carry out biodiversity assessments of these and other sorghums of Pakistan, using SSRs, ISSRs, etc., to further confirm the present findings. The study revealed a narrow genetic base for the local Pakistani sorghum lines, whereas, higher diversity was estimated between local and exotic lines of sorghum. Hence, breeders can improve their efficiency by choosing the diverse parents for sorghum breeding, which may be helpful in broadening the genetic base of the existing local sorghum germplasm of Pakistan.

\section{REFERENCES}

Agrama HA and Tuinstra MR (2003). Phylogenetic diversity and relationships among sorghum accessions using SSRs and RAPDs. Afri. J. Biotech. 2: 334-340.

Amrapali AA, Sakhare SB, Kulwal PL, Dhumale DB, et al. (2008). RAPD profile studies in sorghum for identification of hybrids and their parents. Inter. J. Integ. Bio 3: 18-24.

Anonymous (2008). Pakistan Economic Survey 2007-08. MINFAL, Federal Bureau of Statistics. Islamabad.

Ayana A, Bekele E and Bryngelsson T (2000). Genetic variation in wild sorghum (Sorghum bicolor ssp. verticilliflorum (L.) Moench) germplasm from Ethiopia assessed by random amplified polymorphic DNA (RAPD). Hereditas 132: 249-254.

Baskaran P and Jayabalan N (2005). In vitro plant regeneration and mass propagation system for Sorghum bicolor - a valuable major cereal crop. J. Agri. Tech. 1: 345-363.

Dahlberg JA, Zhang X, Hart GE and Mullet JE (2002). Comparative Assessment of Variation among Sorghum Germplasm Accessions Using Seed Morphology and RAPD Measurements. Crop Sci. 42: 291-296.

Dhillon MK, Sharma HC, Folkertsma RT and Chandra S (2006). Genetic divergence and molecular characterization of sorghum hybrids and their parents for reaction to Atherigona soccata (Rondani). Euphytica 149: 199-210.

Dillon SL, Lawrence PK and Henry RJ (2005). The new use of sorghum bicolor-derived SSR markers to evaluate genetic diversity in 17 Australian sorghum species. Plant Genet. Res. 3: 19-28.

Djè J, Ater M, Lefèbvre C and Vekemans X (1998). Patterns of morphological and allozyme variation in sorghum landraces of Northwestern Morocco. Gen. Res. Crop Evol. 45: 541-548.

Godwin ID and Seetharama N (2005). Sorghum Genetic Engineering: Current Status and Prospectus. In: Sorghum Tissue Culture and Transformation (Seetharama N and Godwin I, eds.). Oxford \& IBH Publishing Co. Pvt. Ltd., New Delhi, 1-8.

Godwin ID, Aitken EA and Smith LW (1997). Application of inter simple sequence repeat (ISSR) markers to plant genetics. Electrophoresis 18: 1524-1528.

Jordan DR, Tao YZ, Godwin ID, Henzell RG, et al. (1998). Loss of genetic diversity associated with selection for 
resistance to sorghum midge in Australian sorghum. Euphytica 102: 1-7.

Kamala V, Bramel PJ, Sivaramakrishnan S, Chandra S, et al. (2006). Genetic and phenotypic diversity in downy-mildewresistant sorghum (Sorghum bicolor (L.) Moench) germplasm. Gen. Res. Crop Evol. 53: 1243-1253.

Khan IA, Awan FS, Ahmed A and Khan AA (2004). A modified mini-prep method for economical and rapid extraction of genomic DNA in plants. Plant Mol. Biol. Reprod. 22: 89a-89e.

Mehmood S, Bashir A, Amad A, Akram Z, et al. (2008). Molecular characterization of regional Sorghum bicolor varieties from Pakistan. Pak. J. Bot. 40: 2015-2021.

Mohamed EA, Youssef SS, Selim EEM and Ghonaim MM (2008). Genetic diversity among Sorghum bicolor genotypes using simple sequence repeats (SSRs) markers. Arab. J. Biotech. 11: 181-192.

Murray SC, Sharma A, Rooney WL, Klein PE, et al. (2008). Genetic improvement of sorghum as a biofuel feedstock: I. QTL for stem sugar and grain nonstructural carbohydrates. Crop Sci. 48: 2165-2179.

Nkongola KK and Nsapato L (2003). Genetic diversity in Sorghum bicolor (L.) Moench accessions from different ecogeographical regions in Malawi assessed with RAPDs. Gen. Res. Crop Evol. 50: 149-156.

Paterson AH, Bowers JE and Chapman BA (2004). Ancient polyploidization predating divergence of the cereals, and its consequences for comparative genomics. Proc. Natl. Acad. Sci. U. S. A. 101: 9903-9908.

Paterson E, Bowers J, Bruggmann R, Inna D, et al. (2009). The Sorghum bicolor genome and the diversification of grasses. Nature 457: 551-553.

Perumal R, Krishnaramanujam R, Menz MA, Katile S, et al. (2007). Genetic diversity among sorghum races and working groups based on AFLPs and SSRs. Crop Sci. 47: 1383.

Rajkumar FB and Kuruvinashetti MS (2007). Genetic variability of sorghum charcoal rot pathogen (Macrophomina phaseolina) assessed by random DNA markers. Plant Pathol. J. 23: 45-50.

Romero G, Adeva C and Battad II Z (2009). Genetic fingerprinting: advancing the frontiers of crop biology research. Phil. Sci. Lett. 2: 8-13.

Shehzad T, Okuizumi H, Kawase M and Okuno K (2009). Development of SSR-based sorghum (Sorghum bicolor (L.) Moench) diversity research set of germplasm and its evaluation by morphological traits. Gen. Res. Crop Evol. 56: 809-827.

Tao Y, Manners JM, Ludlow M and Henzel RJ (1994). DNA polymorphisms in grain sorghum (Sorghum bicolor (L.) Moench). Theor. Appl. Genet. 86: 679-688.

Visarada KBRS and Kishore NS (2007). Improvement of Sorghum through Transgenic Technology. ISB News Report, Rajendranagar, 1-3.

Yeh FC, Yang R, Boyle TJ, Ye Z, et al. (2002). Popgen 32, Microsoftware Windows Based Freeware for Population Genetic Analysis. Molecular Biology and Biotechnology Center, Edmonton. 